\title{
Wheeler-DeWitt equation and Lie symmetries in Bianchi scalar-field cosmology
}

\author{
A. Paliathanasis ${ }^{1}$, L. Karpathopoulos ${ }^{2}$, A. Wojnar ${ }^{3,4,5}$, S. Capozziello ${ }^{4,5,6, a}$ \\ ${ }^{1}$ Instituto de Ciencias Físicas y Matemáticas, Universidad Austral de Chile, Valdivia, Chile \\ ${ }^{2}$ Faculty of Physics, Department of Astronomy-Astrophysics-Mechanics, University of Athens, Panepistemiopolis, 15783 Athens, Greece \\ ${ }^{3}$ Institute for Theoretical Physics, pl. M. Borna 9, 50-204 Wroclaw, Poland \\ ${ }^{4}$ Dipartimento di Fisica “E. Pancini”, Universita' di Napoli Federico II, Complesso Universitario di Monte S. Angelo, Via Cinthia, 9, 80126 Naples, \\ Italy \\ ${ }^{5}$ Istituto Nazionale di Fisica Nucleare (INFN) Sez. di Napoli, Complesso Universitario di Monte S. Angelo, Via Cinthia, 9, 80126 Naples, Italy \\ ${ }^{6}$ Gran Sasso Science Institue (INFN), Viale F. Crispi 7, 67100 L'Aquila, Italy
}

Received: 26 January 2016 / Accepted: 14 April 2016 / Published online: 23 April 2016

(C) The Author(s) 2016. This article is published with open access at Springerlink.com

\begin{abstract}
Lie symmetries are discussed for the Wheeler-De Witt equation in Bianchi Class A cosmologies. In particular, we consider general relativity, minimally coupled scalarfield gravity and hybrid gravity as paradigmatic examples of the approach. Several invariant solutions are determined and classified according to the form of the scalar-field potential. The approach gives rise to a suitable method to select classical solutions and it is based on the first principle of the existence of symmetries.
\end{abstract}

\section{Introduction}

Nowadays astronomical observations have shown that if we consider our Universe on a large scale, its visible structure is accelerating, homogeneous and isotropic, and, essentially, filled with pressureless dust. The simplest cosmic model which describes a Universe with the above properties is the Friedmann-Lemaître-Robertson-Walker (FLRW) model [1]. The evolution of the Universe from the radiation dominant epoch till the present cosmic acceleration can be well explained by the FLRW model with a cosmological constant (the so-called $\Lambda$ CDM model). However, it fails if one tries to address the whole early and late history of the Universe starting from the origin and the inflation epoch where quantum effects should be taken into account.

Anisotropies observed in the cosmic microwave background (CMB) are small enough to suggest that anisotropic models of spacetimes become isotropic ones by evolving in time [2-4]. One can expect that pre-inflationary anisotropies played an important role (for example, they can be respon-

\footnotetext{
a e-mail: capozziello@na.infn.it
}

sible for the coupling between the gravitational field and the inflaton field) so if inflationary models are considered, one should understand the dynamics of anisotropies. Models describing anisotropic but homogeneous Universes are the so-called Bianchi cosmologies. They can be considered in standard general relativity and in its extensions containing scalar fields.

In this paper we will consider the Lie symmetries of the Wheeler-DeWitt equation (WDW) in general relativity and in scalar field cosmology assuming Bianchi spatially homogeneous spacetimes. We will use the Lie symmetries in order to define the unknown potential and derive exact solutions for the WDW equation and for the field equations. Symmetries are considered to play a central role in physical problems because they provide first integrals which can be utilized in order to simplify a given system of differential equations and thus to determine the integrability of the system. Indeed, in $[5,7]$ it has been shown that the Lie symmetries of a dynamical system are related to the geometry of the underlying space where the dynamics occurs.

The application of symmetries in gravitational theories is an important tool which could lead to exact solutions of the field equations. In particular, the symmetries which can be used are the Noether symmetries of the Lagrangian of the field equations and they have been applied in several models such as scalar-tensor cosmology [8-15], $f(R)$ gravity and higher-order theories of gravity [16-21], spherically symmetric spacetimes [22-25], and many others. [26-29]. The application of the Noether symmetries in Bianchi spatially homogeneous spacetimes can be found in [5,30-32]. According to this results one can deal with the so-called Noether symmetry approach. 
In this work we will not apply the Noether symmetries of the field equations but the Lie symmetries of the WDW equation. It has been proved in [7] that the Lie symmetries of the WDW equation could form a greater Lie algebra than the Noether symmetries of the Lagrangian of the field equations. Hence, it is possible to determine new cases where the field equations are integrable. This method was applied in a scalartensor cosmological models adopting a FLRW geometry with a perfect fluid and new integrable models, cosmologically viable, raised [33,34]. Recently a similar method has been applied to some axisymmetric quantum cosmologies with scalar fields [35].

The layout of the paper is the following. In Sect. 2 we give the basic definition of the Bianchi classification while in Sect. 3 we study the Lie symmetries of the WDW equation of Class A Bianchi spacetimes in the case of general relativity. In Sect. 4, the previous results are applied in order to reduce the WDW equation by using the Lie invariants and determine invariant solutions. Sections 5 and 6 are devoted to the same analysis in the case of a minimally coupled scalartensor gravity and we use the Lie symmetries in order to determine the field potential by using the Lie symmetries of the WDW equation as a geometric criterion. We show that, in scalar-tensor cosmology, there exist invariant solutions of the WDW equation: in Bianchi I spacetime, for constant potential and for exponential potential and, in Bianchi II spacetime, for a kination scalar field. For convenience of the reader, we present the Lie symmetry classification for each model in tables. Furthermore in Sect. 7, we show how these results are related with the so-called hybrid gravity and conformal transformations. Finally, in Sect. 8, we discuss our results and draw our conclusions. "Appendix A" completes our presentation. Here the basic theory of Lie symmetries is briefly discussed.

\section{The Class A of Bianchi spacetimes}

The class of Bianchi spatially homogeneous cosmologies contains several important cosmological models which have been used for the discussion of anisotropies of primordial Universe and for its evolution toward the observed isotropy of the present epoch $[2,36,37,39,40]$. In these models, the spacetime manifold is foliated along the time axis, with three dimensional homogeneous hypersurfaces which admit a group of motions $G_{3}$. Bianchi classified all three dimensional real Lie algebras and has shown that there are nine possible $G_{3}$ groups. This results in nine types of Bianchi spatially homogeneous spacetimes. The importance of Bianchi cosmological models is that, in these models, the physical variables depend on the time only, reducing the Einstein and other governing equations to ordinary differential equations.
In the $(3+1)$ decomposition of the spacetime manifold (Arnowitt-Deser-Misner (ADM) formalism), the line element of the Bianchi models can be written in the following form $[41,42]$ :

$\mathrm{d} s^{2}=-\frac{1}{N(t)^{2}} \mathrm{~d} t^{2}+\bar{g}_{i j}(t) \omega^{i} \otimes \omega^{j}$,

where $N(t)$ is the lapse function and $\left\{\omega^{i}\right\}$ denotes the canonical basis 1-forms satisfying the Lie algebra

$d \omega^{i}=C_{j k}^{i} \omega^{j} \wedge \omega^{k}$

where $C_{j k}^{i}$ are the structure constants of the algebra. The spatial metric $\bar{g}_{i j}$ is diagonal (following the notation of $[5,30]$ and references therein) and can be factorized as follows:

$g_{i j}(t)=e^{2 \lambda(t)} e^{-2 \beta_{i j}(t)}$

where $e^{\lambda(t)}$ is the scale factor of the Universe and the matrix $\beta_{i j}$ is diagonal and traceless. The matrix $\beta_{i j}$ depends on two independent quantities $\beta_{1}, \beta_{2}$, which are called the anisotropy parameters. The matrix $\beta_{i j}$ can be selected as ${ }^{1}$

$\beta_{i j}=\operatorname{diag}\left(\beta_{1},-\frac{1}{2} \beta_{1}+\frac{\sqrt{3}}{2} \beta_{2},-\frac{1}{2} \beta_{1}-\frac{\sqrt{3}}{2} \beta_{2}\right)$

and, in these variables, it is $\sqrt{\bar{g}}=e^{3 \lambda}$. The structure constants of the Lie algebra $G_{3}$ can be expressed in terms of a three dimensional vector field $a^{i}$ and a symmetric $3 \times 3$ tensor $m^{i j}$ as follows [43]:

$C_{j k}^{i}=\epsilon_{j k s} m^{s i}+\delta_{k}^{i} a_{j}-\delta_{j}^{i} a_{k}$,

and the Bianchi models are grouped in two classes: Class A for $a^{i}=0$ and Class B for $a^{i} \neq 0$. Each class is divided into several types according to the rank and the signature of the tensor $m^{i j}$. Specifically, the Bianchi models are divided into two subclasses $\mathrm{A}\left(a_{i}=0\right)$ and $\mathrm{B}\left(a_{i} \neq 0\right)$ containing Bianchi types corresponding to the form of the metric $m^{i j}$. In this paper, we are interested in the Class A models for which there exists a Lagrangian of the field equations.

For the line element (1) with the definitions (3) and (4), the Ricci scalar of the Bianchi Class A spacetimes can be written as

$R=R_{(4)}+R^{*}$

where

$R_{(4)}=\frac{3}{2} N\left(4 N \ddot{\lambda}+4 \dot{N} \dot{\lambda}+8 N \dot{\lambda}^{2}+N \dot{\beta}_{1}^{2}+N \dot{\beta}_{2}^{2}\right)$

and $R^{*}=R^{*}\left(\lambda, \beta_{1}, \beta_{2}\right)$ is the component of the three dimensional hypersurface which depends on the structure constants of the algebra $N_{1-3}$ of the Killing algebra of the Bianchi algebras [44]. The general form of $R^{*}$ is

\footnotetext{
1 These are the Misner variables [42].
} 


$$
\begin{aligned}
R^{*}= & -\frac{1}{2} e^{-2 \lambda}\left[\begin{array}{c}
N_{1}^{2} e^{4 \beta_{1}}+e^{-2 \beta_{2}}\left(N_{2} e^{\sqrt{3} \beta_{2}}-N_{3} e^{-\sqrt{3} \beta_{2}}\right)^{2}+ \\
-2 N_{1} e^{\beta_{1}}\left(N_{2} e^{\sqrt{3} \beta_{2}}-N_{3} e^{-\sqrt{3} \beta_{2}}\right)^{2}
\end{array}\right] \\
& +\frac{1}{2} N_{1} N_{2} N_{3}\left(1+N_{1} N_{2} N_{3}\right)
\end{aligned}
$$

and the special forms for the Class A spacetimes can be found in Table 1.

In the case of general relativity, when the action of the field equations is the Einstein-Hilbert action (we consider the spacetime to be empty)

$S_{G R}=\int \mathrm{d} x^{4} \sqrt{-g} R$,

the field equations for the Bianchi Class A spacetimes follow from the Lagrangian

$$
\begin{aligned}
& L\left(N, \lambda, \beta_{1}, \beta_{2}, \dot{\lambda}, \dot{\beta}_{1}, \dot{\beta}_{2}\right) \\
& \quad=N(t) e^{3 \lambda}\left(6 \dot{\lambda}^{2}-\frac{3}{2}\left(\dot{\beta}_{1}^{2}+\dot{\beta}_{2}^{2}\right)\right)+\frac{e^{3 \lambda}}{N(t)} R^{*},
\end{aligned}
$$

and the corresponding field equations are the Euler-Lagrange equations with respect to the variables $\left\{N, \lambda, \beta_{1}, \beta_{2}\right\}$. The Euler-Lagrange equations for the variables $\beta_{1}, \beta_{2}$ are

$\ddot{\beta}_{(1,2)}+\frac{\dot{N}}{N} \dot{\beta}_{(1,2)}+3 \dot{\lambda} \dot{\beta}_{(1,2)}+\frac{1}{3 N^{2}} R_{,(1,2)}^{*}=0 ;$

for the variable $\lambda$

$$
\begin{aligned}
4 \ddot{\lambda} & +\left(6 \dot{\lambda}^{2}+\frac{3}{2}\left({\dot{\beta_{1}}}^{2}+{\dot{\beta_{2}}}^{2}\right)+\frac{1}{2} \dot{\phi}^{2}\right) \\
& +\frac{\dot{N}}{N} \dot{\lambda}-\frac{1}{N^{2}}\left(R^{*}+\frac{1}{3} \frac{\partial R^{*}}{\partial \lambda}\right)=0,
\end{aligned}
$$

and for the variable $N$ we have the $G_{0}^{0}=0$ Einstein equation

$$
N(t) e^{3 \lambda}\left(6 \dot{\lambda}^{2}-\frac{3}{2}\left(\dot{\beta}_{1}^{2}+\dot{\beta}_{2}^{2}\right)\right)-\frac{e^{3 \lambda}}{N(t)} R^{*}=0 .
$$

The Lagrangian (10) is singular since $\frac{\partial L}{\partial \dot{N}}=0$, however, if we consider

$N(t)=N_{0} \quad$ or $\quad N(t)=N\left(\lambda(t), \beta_{1}(t), \beta_{2}(t)\right)$

then the Lagrangian (10) becomes a regular time independent Lagrangian which admits always, as a Noether integral, the Hamiltonian constant. Hence, Eq. (13) can be seen as the energy constraint of the field equations.

In the following we will quantize Eq. (13) in order to write the Wheeler-DeWitt (WDW) equation and to perform a symmetry analysis using the Lie point symmetries in the case of general relativity and minimally coupled scalar-tensor cosmology.

\section{Symmetries of the WDW equation in general relativity}

In order to simplify Eq. (13), we consider $N(t)=\bar{N}(t) e^{-3 \lambda}$ in the line element (1). Furthermore, we consider the change of the variables $\left(\lambda, \beta_{1}, \beta_{2}\right)=\left(\frac{\sqrt{3}}{6} x, \frac{\sqrt{3}}{3} y, \frac{\sqrt{3}}{3} z\right)$, then the Lagrangian (10) becomes

$L=\frac{1}{2} \bar{N}\left(\dot{x}^{2}-\dot{y}^{2}-\dot{z}^{2}\right)+\frac{1}{\bar{N}} e^{\sqrt{3} x} R^{*}$.

Therefore, we define the momentum $p_{(x, y, z)}=\frac{\partial L}{\partial(\dot{x}, \dot{y}, \dot{z})}$ and Eq. (13) has now the form

$\frac{1}{2}\left(p_{x}^{2}-p_{y}^{2}-p_{z}^{2}\right)-e^{\sqrt{3} x} R^{*}=0$.

Equation (15) can be seen as the Hamiltonian of a particle moving in the space $M^{3}$ with potential $V(x, y, z)=$ $-e^{\sqrt{3} x} R^{*}$. Furthermore, the field equations are the Hamiltonian constraint (15) and the Hamilton equations

$\dot{x}=\frac{1}{\bar{N}} p_{x}, \quad \dot{y}=-\frac{1}{\bar{N}} p_{y}, \dot{z}=-\frac{1}{\bar{N}} p_{z}$

$\dot{p}_{x}=-\sqrt{3}\left(1+\left(\ln R^{*}\right)_{x}\right) e^{\sqrt{3} x} R^{*}$,

$\dot{p}_{y}=e^{\sqrt{3} x} R_{, y}^{*}, \quad \dot{p}_{z}=e^{\sqrt{3} x} R_{, z}^{*}$.

Since the minisuperspace is flat, that is, the Ricci scalar vanishes, the WDW equation can be achieved by a standard quantization, assuming the conjugate momenta $p_{i}=\frac{\partial L}{\partial x^{i}}$. Hence from (15), we have the WDW equation of the form

$\Psi_{, x x}-\Psi_{, y y}-\Psi_{, z z}-2 e^{\sqrt{3} x} R^{*} \Psi=0$,

which is nothing else but the Klein-Gordon equation in the $M^{3}$ space.

In order to determine the Lie symmetries of (19), we will use the geometric results in Ref. [7]. The $M^{3}$ spacetime admits a ten dimensional conformal algebra. In particular, it admits a six dimensional Killing algebra which is the $T^{3} \cup S O$ (3) with elements

$K_{(x)}=\partial_{x}, \quad K_{(y)}=\partial_{y}, \quad K_{(z)}=\partial_{z}$,

$R_{(x y)}=y \partial_{x}+x \partial_{y}, \quad R_{(x z)}=z \partial_{x}+x \partial_{z}, \quad R_{(y z)}=z \partial_{y}-y \partial_{z}$,

one gradient homothetic Killing vector (HV),

$H=x \partial_{x}+y \partial_{y}+z \partial_{z}, \quad \psi_{H}=1$,

and three special conformal Killing vectors (CKVs), which are

$$
\begin{array}{ll}
C_{(x)}=\frac{1}{2}\left(x^{2}+y^{2}+z^{2}\right) \partial_{x}+x y \partial_{y}+x z \partial_{z}, & \psi_{(x)}=x, \\
C_{(y)}=x y \partial_{x}+\frac{1}{2}\left(x^{2}+y^{2}-z^{2}\right) \partial_{y}+z y \partial_{z}, & \psi_{(y)}=y, \\
C_{(z)}=x z \partial_{x}+y z \partial_{y}+\frac{1}{2}\left(x^{2}+z^{2}-y^{2}\right) \partial_{z}, & \psi_{(z)}=z .
\end{array}
$$


Table 1 The Ricci scalar of the $3 \mathrm{~d}$ hypersurfaces of the Class A Bianchi spacetimes

\begin{tabular}{ll}
\hline Model & $R^{*}\left(\lambda, \beta_{1}, \beta_{2}\right)$ \\
\hline Bianchi I & 0 \\
Bianchi II & $-\frac{1}{2} e^{\left(4 \beta_{1}-2 \lambda\right)}$ \\
Bianchi VI /VII $_{0}$ & $-\frac{1}{2} e^{-2 \lambda}\left(e^{4 \beta_{1}}+e^{-2\left(\beta_{1}-\sqrt{3} \beta_{2}\right)} \pm 2 e^{\beta_{1}+\sqrt{3} \beta_{2}}\right)$ \\
Bianchi VIII & $-\frac{1}{2} e^{-2 \lambda}\left(\begin{array}{c}e^{4 \beta_{1}}+e^{-2 \beta_{1}}\left(e^{\sqrt{3} \beta_{2}}+e^{-\sqrt{3} \beta_{2}}\right)^{2}+ \\
-2 e^{-\beta_{1}}\left(e^{\sqrt{3} \beta_{2}}-e^{-\sqrt{3} \beta_{2}}\right)^{2}\end{array}\right)$ \\
Bianchi IX & $-\frac{1}{2} e^{-2 \lambda}\left(\begin{array}{c}e^{4 \beta_{1}}+e^{-2 \beta_{1}}\left(e^{\sqrt{3} \beta_{2}}-e^{-\sqrt{3} \beta_{2}}\right)^{2}+ \\
-2 e^{-\beta_{1}}\left(e^{\sqrt{3} \beta_{2}}+e^{-\sqrt{3} \beta_{2}}\right)^{2}\end{array}\right)+1$ \\
\hline
\end{tabular}

See Ref. [7] for details. Furthermore, by applying the results in [7], we find that the WDW equation (19) admits: (1) for the Bianchi I model, 11 Lie symmetries, (2) for the Bianchi II model, five Lie symmetries, (3) two Lie symmetries for the models $\mathrm{VI}_{0} / \mathrm{VII}_{0}$, and (4) one Lie symmetry, the linear one, for the models VIII and IX. In Table 2, we give the corresponding Lie symmetries of the WDW equation (19) for each Bianchi model.

\section{Invariant solutions of the WDW equation in general relativity}

In this section, we will apply the zeroth-order invariants of the Lie point symmetries to reduce the order of the WDW equation (19) and to determine invariant solutions. An important remark is due at this point. In general, invariant solutions are defined as the solutions following from the application of invariant functions of an admitted symmetry vector for the given differential equation. ${ }^{2}$ In other words [38], a solution $\Psi=\Psi\left(x_{i}\right), x_{i}=x, y, \ldots$, is invariant under a group generated by the vector $X=\xi_{i} \partial_{x^{i}}+\eta \partial_{\Psi}$, if and only if the characteristic vanishes on the solution, that is, an invariant solution satisfies the invariant $n$-surface condition ( $n$ being the number of independent variables):

$\eta-\xi^{i} \partial_{x^{i}} \Psi=0$

We will see that solutions given in the following sections satisfy the above condition.

From the results of Table 2, we can see that it is possible to find invariant solutions for the WDW equation for Bianchi I and II spacetimes.

$\overline{{ }^{2} \text { For details on }}$ the application of Lie symmetries see "Appendix A".

\subsection{Bianchi I cosmology}

Since for Bianchi I spacetimes the property holds that $R^{*}=$ 0 , the WDW equation (19) is the $(1+2)$ wave equation. The reduction of the $(1+2)$ wave equation and the invariant solutions have been studied in [45] and recently in [46]. However, we want to give a concrete example of the application of the Lie symmetries.

Let us consider the Lie algebra $\left\{X_{(x)}^{I}, X_{(y)}^{I}\right\}$ with commutators $\left[X_{(x)}^{I}, X_{(y)}^{I}\right]=0$ where

$X_{\left(x^{i}\right)}^{I}=K_{\left(x^{i}\right)}+\mu_{i} \Psi \partial_{\Psi}$

Consider the Lie symmetry vector $X_{(x)}^{I}=\partial_{x}+\mu_{1} \Psi \partial_{\Psi}$, from which we find that the zeroth-order Lie invariants are $\left\{y, z, \exp \left(-\mu_{1} x\right) \Psi\right\}$. Considering now as $\Psi=\exp$ $\left(\mu_{1} x\right) \bar{\Psi}(y, z)$, where $\bar{\Psi}$ is the new dependent variable, then Eq. (19) reduce to the two-dimensional linear Klein-Gordon equation

$\mu_{1}^{2} \bar{\Psi}-\bar{\Psi}_{, y y}-\bar{\Psi}_{, z z}=0$.

If we continue with the application of the Lie invariants of the symmetry vectors $X_{(y)}^{I}$ and $X_{(z)}^{I}$, then we find following solution:

$$
\begin{aligned}
\Psi_{I}(x, y, z)= & \exp \left(\mu_{1} x+\mu_{2} y\right)\left[c_{1} \exp \left(\sqrt{\mu_{1}^{2}-\mu_{2}^{2}} z\right)\right. \\
& \left.+c_{2} \exp \left(-\sqrt{\mu_{1}^{2}-\mu_{2}^{2}} z\right)\right]
\end{aligned}
$$

From this one can see that (21) is invariant under the point transformation with generators the vector fields (20).

One can also consider the Lie algebra $\left\{X_{(x)}^{I}, X_{(y z)}^{I}=\right.$ $\left.R_{(y z)}+\mu \Psi \partial_{\Psi}\right\}$ for which the invariant solution is 
Table 2 Lie symmetries of the WDW equation of the Class A Bianchi models in general relativity

\begin{tabular}{lll}
\hline Model & $\#$ & Lie Symmetries \\
\hline Bianchi I & 11 & $\begin{array}{l}\Psi \partial_{\Psi}, K_{(x)}, K_{(y)}, K_{(z)}, R_{(x y)}, R_{(x z)}, R_{(y z)}, H \\
\left(C_{(x)}-\frac{1}{2} x \Psi \partial_{\Psi}\right),\left(C_{(y)}-\frac{1}{2} y \Psi \partial_{\Psi}\right),\left(C_{(z)}-\frac{1}{2} z \Psi \partial_{\Psi}\right)\end{array}$ \\
Bianchi II & 4 & $\Psi \partial_{\Psi}, K_{(z)}, K_{(x)}-\frac{1}{2} K_{(y)}, R_{(x z)}-\frac{1}{2} R_{(y z)}$ \\
Bianchi VI $/ \mathrm{VII}_{0}$ & 2 & $\Psi \partial_{\Psi}, K_{(x)}-\frac{1}{2} K_{(y)}+\frac{\sqrt{3}}{2} K_{(z)}$ \\
Bianchi VIII/IX & 1 & $\Psi \partial_{\Psi}$ \\
\hline
\end{tabular}

$$
\begin{aligned}
& \Psi_{I}(x, y, z) \\
& =\left[c_{1} I_{i \mu}\left(\mu_{1} \sqrt{y^{2}+z^{2}}\right)+c_{2} K_{i \mu}\left(\left(\mu_{1} \sqrt{y^{2}+z^{2}}\right)\right)\right] \\
& \quad \times \exp \left(\mu \arctan \frac{y}{z}+\mu_{1} x\right)
\end{aligned}
$$

where $I_{i \mu}, K_{i \mu}$ are the modified Bessel functions of the first and the second kinds. Similarly, for the others Lie subalgebras, we can construct invariant solutions. We want also to recall that the WDW equation is a linear second-order partial differential equation and any linear combination of the solutions is also a solution.

\subsubsection{The WKB approximation and the classical solution}

Adopting the WKB approximation to the WDW equation ( $\Psi \simeq \exp (i S)$ ), Eq. (19) becomes

$$
\left(\frac{\partial S}{\partial x}\right)^{2}-\left(\frac{\partial S}{\partial y}\right)^{2}-\left(\frac{\partial S}{\partial z}\right)^{2}=0
$$

which is the null Hamilton-Jacobi equation of the Hamiltonian system (15)-(18), where $p_{i}=\frac{\partial S}{\partial x^{i}}$. The solution of (23) is

$S(x, y, z)=s_{1} y+s_{2} z+\varepsilon \sqrt{s_{1}^{2}+s_{2}^{2}} x+s_{0}, \quad$ with $\quad \varepsilon= \pm 1$.

Hence the Hamilton equations (16)-(18) reduce to the following system (where $\bar{N}=1$ ):

$\dot{x}=\varepsilon \sqrt{s_{1}^{2}+s_{2}^{2}}, \quad \dot{y}=-s_{1}, \quad \dot{z}=-s_{2}$

and therefore we have the solutions

$$
\left(\lambda(t), \beta_{1}(t), \beta_{2}(t)\right)=\left(\frac{\sqrt{3}}{6} \varepsilon \sqrt{s_{1}^{2}+s_{2}^{2}} t,-\frac{\sqrt{3}}{3} s_{1} t,-\frac{\sqrt{3}}{3} s_{2} t\right)
$$

and, by the coordinate transformation $\mathrm{d} \tau=e^{3 \lambda} \mathrm{d} t$ in the line element (1), we obtain a Kasner spacetime. Let us note that Kasner parameters $s_{1}, s_{2}$, are related to the constants $\mu_{1}, \mu_{2}$ and correspond to the oscillatory terms of the solution (21).

\subsection{Bianchi II cosmology}

For the Bianchi II spacetime, using Table 2, we see that if we use the Lie symmetries $\left\{X_{(z)}^{I}, X_{(x)}^{I}-\frac{1}{2} X_{(y)}^{I}\right\}$ with zero commutator, the invariant solution is

$$
\begin{aligned}
\Psi_{I I}^{1}(x, y, z)= & \exp \left(\frac{2 \mu_{12}}{3}(y+2 x)+\mu_{3} z\right) \\
& \times\left(c_{1} I_{c_{0}}(u(x, y))+c_{2} K_{c_{0}}(u(x, y))\right)
\end{aligned}
$$

where $\mu_{12}=\mu_{1}-\mu_{2}, c_{0}=\frac{\sqrt{12 \mu_{12}^{2}-9 \mu_{3}^{2}}}{3}, u(x, y)=$ $\exp \left(\frac{\sqrt{3}(2 y+x)}{3}\right)$, and $I_{c_{0}}, K_{c_{0}}$ are the modified Bessel functions of the first and the second kinds, respectively. For the Lie algebra

$K_{(x)}^{I}-\frac{1}{2} K_{(y)}^{I}, \quad R_{(x z)}-\frac{1}{2} R_{(y z)}$

we have the invariant solution

$\Psi_{I I}^{2}(x, y, z)=\left(c_{1} I_{0}(u(x, y))+c_{2} K_{0}(u(x, y))\right)$.

The last solution $\Psi_{I I}^{2}$ is included in the solution $\Psi_{I I}^{1}$ for $\mu_{12}=\mu_{3}=0$.

\subsubsection{The WKB approximation and the classical solution}

One can also apply the WKB approximation for the Bianchi II model to Eq. (19):

$$
\left(\frac{\partial S}{\partial x}\right)^{2}-\left(\frac{\partial S}{\partial y}\right)^{2}-\left(\frac{\partial S}{\partial z}\right)^{2}+e^{\frac{2 \sqrt{3}}{3}(2 y+x)}=0
$$

Let us adopt, the coordinate transformation $y=w-\frac{x}{2}$ in the Hamiltonian system. Hence, the new Hamilton-Jacobi equation (29) becomes

$$
\left(\frac{\partial S}{\partial x}\right)^{2}+\left(\frac{\partial S}{\partial x}\right)\left(\frac{\partial S}{\partial w}\right)-\frac{3}{4}\left(\frac{\partial S}{\partial w}\right)^{2}-\left(\frac{\partial S}{\partial z}\right)^{2}+e^{\frac{4 \sqrt{3}}{3} w}=0,
$$

with the solution

$S(x, w, z)=S_{1}(x)+S_{2}(w)+S_{3}(z)$, 
where the functions $S_{1,2,3}$ follow from the system

$$
S_{1}(x)=c_{1} x, \quad S_{3}(z)=c_{2} z,
$$

$$
\left(\frac{\mathrm{d} S_{2}}{\mathrm{~d} w}\right)^{2}=\frac{4}{3}\left[c_{1}\left(c_{1}+\frac{\mathrm{d} S_{2}}{\mathrm{~d} w}\right)+c_{2}^{2}-2 e^{\frac{4 \sqrt{3}}{3} w}\right]=0 .
$$

The Hamilton function $S(x, w, z)$ is

$$
\begin{aligned}
& S(x, w, z)=c_{1} x+c_{2} z+\frac{2}{3} c_{1} w-\varepsilon \frac{\sqrt{3}}{3} \\
& \quad \times\left(M(w)-\sqrt{c_{12}} \arctan \left(\frac{M(w)}{\sqrt{c_{12}}}\right)\right), \quad \text { with } \varepsilon= \pm 1
\end{aligned}
$$

where $c_{12}=3 c_{2}^{2}-4 c_{1}^{2}$ and $M(w)=\sqrt{3 e^{\frac{4 \sqrt{3}}{3} w}-c_{12}}$. Therefore the reduced Hamiltonian system is (recall that $\bar{N}=1)$

$\dot{z}=-2 c_{2}, \quad \dot{w}=\varepsilon M(w)$

$\dot{x}=\frac{8}{3} c_{1}-\varepsilon \frac{2}{3} M(w)$

Hence the solution of the system (35), (36) is

$$
\begin{aligned}
& z(t)=-2 c_{2} t \\
& w(t)=-\frac{\sqrt{3}}{4} \ln \left(\frac{\cos ^{2}\left(\frac{2 \sqrt{3}}{3} \varepsilon \sqrt{c_{12}}\left(t+w_{0}\right)\right)}{c_{12}}\right) \\
& x(t)=\frac{\sqrt{3}}{6} \ln \left[\cos ^{2}\left(\frac{2 \sqrt{3}}{3} \varepsilon \sqrt{c_{12}}\left(t+w_{0}\right)\right)\right]+\frac{8}{3} c_{1} t+x_{0}
\end{aligned}
$$

which is the solution of the empty Bianchi II spacetime in general relativity.

In the following section we will apply the same procedure in the case of minimally coupled scalar-tensor cosmology. Furthermore, we will use the Lie symmetries of the WDW equation in order to determine the unknown potential of the scalar field.

\section{Symmetries of the WDW equation in minimally coupled scalar-tensor cosmology}

Let us continue the Lie symmetry analysis of the WDW equation for cosmological containing a minimally coupled scalar field in the gravitational action. The Noether symmetry classification of the field equations has been studied in [30-32]. Noether symmetries have been adopted in the jet space in [5]. In Ref. [6], a detailed study of integrable cosmological models with non-minimally coupled scalar fields is presented.
Let us now take into account the following action:

$S=\int \mathrm{d} x^{4} \sqrt{-g}\left(R-\frac{1}{2} g^{\mu v} \phi_{, \mu} \phi_{\nu}+V(\phi)\right)$.

From the line element (1) and equations (6), (7) we find the Lagrangian [36]

$$
\begin{aligned}
& L\left(N, \lambda, \beta_{1}, \beta_{2}, \phi, \dot{\lambda}, \dot{\beta}_{1}, \dot{\beta}_{2}, \dot{\phi}\right) \\
& =N(t) e^{3 \lambda}\left(6 \dot{\lambda}^{2}-\frac{3}{2}\left(\dot{\beta}_{1}^{2}+\dot{\beta}_{2}^{2}\right)-\frac{1}{2} \dot{\phi}^{2}\right) \\
& \quad+\frac{e^{3 \lambda}}{N(t)}\left(V(\phi)+R^{*}\right) .
\end{aligned}
$$

Hence, by applying the Euler-Lagrange vector in (41), we find four field equations which are the two equations (11) and

$$
\begin{aligned}
4 \ddot{\lambda} & +\left(6 \dot{\lambda}^{2}+\frac{3}{2}\left(\dot{\beta}_{1}{ }^{2}+\dot{\beta}_{2}{ }^{2}\right)+\frac{1}{2} \dot{\phi}^{2}\right) \\
& +\frac{\dot{N}}{N} \dot{\lambda}-\frac{1}{N^{2}}\left(V+R^{*}+\frac{1}{3} \frac{\partial R^{*}}{\partial \lambda}\right)=0,
\end{aligned}
$$

$N e^{3 \lambda}\left(6 \dot{\lambda}^{2}-\frac{3}{2}\left(\dot{\beta}_{1}^{2}+\dot{\beta}_{2}^{2}\right)-\frac{1}{2} \dot{\phi}^{2}\right)-\frac{e^{3 \lambda}}{N}\left(V+R^{*}\right)=0$.

The latter corresponds to the $G_{0}^{0}=T_{0}^{0}$ Einstein equation. Furthermore, from the Euler-Lagrange equation $\frac{\mathrm{d}}{\mathrm{d} t}\left(\frac{\partial L}{\partial \dot{\phi}}\right)$ $-\frac{\partial L}{\partial \phi}$, we obtain the field equation for the scalar field

$\ddot{\phi}+3 \dot{\lambda} \dot{\phi}+\frac{\dot{N}}{N} \dot{\phi}+\frac{1}{N^{2}} \frac{\partial V}{\partial \phi}=0$,

which can also be derived by the Bianchi identity $T_{; \nu}^{\mu \nu}=0$, where $T^{\mu \nu}$ is the energy-momentum tensor for scalar field. As in the case of general relativity, the coordinate transformations $\left(\lambda, \beta_{1}, \beta_{2}\right)=\left(\frac{\sqrt{3}}{6} x, \frac{\sqrt{3}}{3} y, \frac{\sqrt{3}}{3} z\right)$ and $N(t)=$ $\bar{N}(t) e^{-3 \lambda}$, can be adopted. Hence, Eq. (43) becomes

$\frac{1}{2} \bar{N}\left(\dot{x}^{2}-\dot{y}^{2}-\dot{z}^{2}-\dot{\phi}^{2}\right)-\frac{1}{\bar{N}} e^{\sqrt{3} x}\left(V(\phi)+R^{*}\right)=0$.

Moreover, by using the momentum $p_{(x, y, z, \phi)}=\frac{\partial L}{\partial x^{i}}$, equation (45) becomes

$\frac{1}{2}\left(p_{x}^{2}-p_{y}^{2}-p_{z}^{2}-p_{\phi}^{2}\right)-e^{\sqrt{3} x}\left(V(\phi)+R^{*}\right)=0$

and hence, from (46), we have the following WDW equation:

$\Psi_{, x x}-\Psi_{, y y}-\Psi_{, z z}-\Psi_{, \phi \phi}-2 e^{\sqrt{3} x}\left(V(\phi)+R^{*}\right) \Psi=0$, 
since the minisuperspace of Eq. (46) is the flat space $M^{4}$. We note that Eq. (47) is the Klein-Gordon equation in $M^{4}$.

Since we want to use the geometric approach in Ref. [7], we need the conformal algebra of the $M^{4}$ spacetime. The four dimensional flat space $M^{4}$ admits ten Killing vectors (KVs) which are

$$
\begin{aligned}
& K_{(x)}, K_{(y)}, K_{(z)}, K_{(\phi)}=\partial_{\phi}, \\
& R_{(x y)}, R_{(x z)}, R_{(y z)}, R_{(x \phi)}=\phi \partial_{x}+x \partial_{\phi}, \\
& R_{(y \phi)}=\phi \partial_{y}-y \partial_{\phi}, \quad R_{(z \phi)}=\phi \partial_{z}-z \partial_{\phi},
\end{aligned}
$$

\section{one gradient $\mathrm{HV}$}

$\bar{H}=x \partial_{x}+y \partial_{y}+z \partial_{z}+\phi \partial_{\phi}$,

and four special CKVs

$$
\begin{aligned}
\bar{C}_{(x)}= & C_{(x)}+\frac{1}{2} \phi^{2} \partial_{x}+\phi x \partial_{\phi}, \quad \psi_{(x)}=x, \\
\bar{C}_{(y)}= & C_{(y)}-\frac{1}{2} \phi^{2} \partial_{y}+\phi y \partial_{\phi}, \quad \psi_{(y)}=y, \\
\bar{C}_{(z)}= & C_{(z)}-\frac{1}{2} \phi^{2} \partial_{z}+\phi z \partial_{\phi}, \quad \psi_{(z)}=z, \\
\bar{C}_{(\phi)}= & x \phi \partial_{x}+y \phi \partial_{y}+z \phi \partial_{z} \\
& +\frac{1}{2}\left(x^{2}+\phi^{2}-y^{2}-z^{2}\right) \partial_{\phi}, \quad \psi_{(\phi)}=\phi .
\end{aligned}
$$

One can find that the WDW equation (47) admits Lie symmetries for an arbitrary potential $V(\phi)$; in the case of a Bianchi I, spacetime Eq. (47) admits four Lie symmetries; for Bianchi II, one has two Lie symmetries and one Lie symmetry for the rest of the Bianchi models. Therefore, for special forms of the potentials $V(\phi)$, it is possible that the WDW equation (47) admits extra symmetries. The special forms of the potentials that we found are: (a) $V(\phi)=0$, in which the scalar field behaves as stiff matter; (b) $V(\phi)=V_{0}$ with $V_{0} \neq 0$, and (c) $V(\phi)=V_{0} e^{\mu \phi}$. The results of the symmetry analysis are collected in Tables 3 and 4.
We will continue our analysis using the results of Tables 3 and 4 in order to determine invariant solutions of the WDW equation (47) for cases where it is possible.

\section{Invariant solutions of the WDW equation in scalar-field cosmology}

From the symmetries in Tables 3 and 4, we observe that invariant solutions for the WDW equation can be determined for the Bianchi type I model for (a) $V(\phi)=0$, (b) $V(\phi)=$ $V_{0}$, (c) $V(\phi)=V_{0} e^{\mu \phi}$; and, for the Bianchi II model, for zero potential. It is worth noticing that, in the following, we will choose $\bar{N}(t)=1$.

\subsection{Bianchi I cosmology}

For the Bianchi I spacetime, the WDW equation (47) becomes

$$
\Psi_{, x x}-\Psi_{, y y}-\Psi_{, z z}-\Psi_{, \phi \phi}-2 e^{\sqrt{3} x} V(\phi) \Psi=0 .
$$

If the potential $V(\phi)=0$, then (48) becomes the $(1+3)$ wave equation in $E^{3}$ [45], hence we will omit this case. When $V(\phi)=V_{0}, V_{0} \neq 0$, the field equations are equivalent to the case of general relativity with stiff matter and a cosmological constant. In this case, we use zeroth-order invariants of the Lie symmetries

$\bar{X}_{(A)}=K_{(A)}+\mu_{(A)} \Psi \partial_{\Psi}, \quad A=y, z, \phi$,

which form a closed Lie algebra. In this case, Eq. (48) reduces to the linear second-order ordinary differential equation

$\Phi^{\prime \prime}-\left(\mu_{(y)}+\mu_{(z)}+\mu_{(z)}+2 V_{0} e^{\sqrt{3} x}\right) \Phi=0$

where $\Psi=\Phi(x) \exp \left(\mu_{(y)} y+\mu_{(z)} z+\mu_{(\phi)} \phi\right)$ and $\Phi^{\prime}=$ $\frac{\mathrm{d} \Phi(x)}{\mathrm{d} x}$. Therefore, the solution of Eq. (50) is

$$
\Phi(x)=\Phi_{1} J_{c}\left(i \frac{2 \sqrt{6 V_{0}}}{3} e^{\frac{\sqrt{3}}{2} x}\right)+\Phi_{2} Y_{c}\left(i \frac{2 \sqrt{6 V_{0}}}{3} e^{\frac{\sqrt{3}}{2} x}\right)
$$

Table 3 Lie symmetries of the WDW equation of the Class A Bianchi models in scalar-field cosmology for $V(\phi)=0$

\begin{tabular}{lll}
\hline Model $V(\phi)=0$ & $\#$ & Lie symmetries \\
\hline Bianchi I & 16 & $\Psi \partial_{\Psi}, K_{(x)}, K_{(y)}, K_{(z)}, K_{(\phi)}, R_{(x y)}, R_{(x z)}$ \\
& $R_{(y z)}, R_{(x \phi)}, R_{(y \phi)}, R_{(z \phi)}, \bar{H},\left(\bar{C}_{(x)}-x \Psi \partial_{\Psi}\right)$, \\
& $\left(\bar{C}_{(y)}-y \Psi \partial_{\Psi}\right),\left(\bar{C}_{(z)}-z \Psi \partial_{\Psi}\right),\left(\bar{C}_{(\phi)}-\phi \Psi \partial_{\Psi}\right)$ \\
Bianchi II & $\Psi \partial_{\Psi}, K_{(z)}, K_{(\phi)}, K_{(x)}-\frac{1}{2} K_{(y)}, R_{(z \phi)}$ \\
& 7 & $R_{(x z)}-\frac{1}{2} R_{(y z)}, R_{(x \phi)}-\frac{1}{2} R_{(y \phi)}$ \\
Bianchi VI ${ }_{0} / \mathrm{VII}_{0}$ & 3 & $\Psi \partial_{\Psi}, K_{(\phi)}, K_{(x)}+\frac{1}{4} K_{(y)}+\frac{\sqrt{3}}{4} K_{(z)}$ \\
& & $R_{(x \phi)}+\frac{1}{4} R_{(y \phi)}+\frac{\sqrt{3}}{4} R_{(z \phi)}$ \\
Bianchi VIII/IX & & $\Psi \partial_{\Psi}, K_{(\phi)}$ \\
\hline
\end{tabular}


Table 4 Lie symmetries of the WDW equation of the Class A Bianchi models in scalar-field cosmology for non-zero potentials

\begin{tabular}{lll}
\hline Model $V(\phi)=V_{0}$ & $\#$ & Lie symmetries \\
Bianchi I & 7 & $\Psi \partial_{\Psi}, K_{(y)}, K_{(z)}, K_{(\phi)}, R_{(y z)}, R_{(y \phi)}, R_{(z \phi)}$ \\
Bianchi II & 4 & $\Psi \partial_{\Psi}, K_{(z)}, K_{(\phi)}, R_{(z \phi)}$ \\
Bianchi VI $/ \mathrm{VII}_{0}$ & 2 & $\Psi \partial_{\Psi}, K_{(\phi)}$ \\
Bianchi VIII/IX & 2 & $\Psi \partial_{\Psi}, K_{(\phi)}$ \\
Model $V(\phi)=V_{0} e^{\mu \phi}$ & $\#$ & Lie symmetries \\
Bianchi I & 7 & $\Psi \partial_{\Psi}, K_{(y)}, K_{(z)}, R_{(y z)}, \frac{\sqrt{3}}{3} \mu K_{(x)}-K_{(\phi)}$, \\
& & $R_{(y \phi)}+\frac{\sqrt{3}}{3} \mu R_{(x y)}, R_{(z \phi)}+\frac{\sqrt{3}}{3} \mu R_{(x z)}$ \\
Bianchi II & $\Psi \partial_{\Psi}, K_{(z)}, K_{(x)}-\frac{1}{2} K_{(y)}-\frac{\sqrt{3}}{\mu} K_{(\phi)}$ \\
& 4 & $R_{(z \phi)}+\frac{\sqrt{3}}{3} \mu\left(R_{(x z)}-\frac{1}{2} R_{(y z)}\right)$ \\
Bianchi VI $/ \mathrm{VII}{ }_{0}$ & & $\Psi \partial_{\Psi}, K_{(x)}-\frac{1}{2} K_{(y)}-\frac{\sqrt{3}}{2} K_{(z)}-\frac{\sqrt{3}}{\mu} K_{(\phi)}$ \\
Bianchi VIII/IX & 2 & $\Psi \partial_{\Psi}$ \\
Model $V(\phi)=V(\phi)$ & 1 & Lie symmetries \\
Bianchi I & $\#$ & $\Psi \partial_{\Psi}, K_{(y)}, K_{(z)}, R_{(y z)}$ \\
Bianchi II & 4 & $\Psi \partial_{\Psi}, K_{(z)}$ \\
Bianchi VI $/ \mathrm{VII} 0$ & $\Psi \partial_{\Psi}$ \\
Bianchi VIII/IX & 2 & $\Psi \partial_{\Psi}$ \\
\hline
\end{tabular}

where $J_{c}, Y_{c}$ are the Bessel functions of the first and second kind and the constant is $c=\frac{2 \sqrt{3}}{3}\left(\sqrt{\mu_{(y)}^{2}+\mu_{(z)}^{2}+\mu_{(z)}^{2}}\right)$. For the exponential potential, we apply the zeroth-order invariants of the Lie symmetries

$\bar{X}_{(y)}, \bar{X}_{(z)}, \frac{\sqrt{3}}{3} \mu K_{(x)}-K_{(\phi)}+v \Psi \partial_{\Psi}$

and the WDW equation (48) becomes

$$
\begin{aligned}
& \left(3-\mu^{2}\right) \Phi^{\prime \prime}(w)+6 v \Phi^{\prime}-\left(\left(\mu_{(y)}^{2}+\mu_{(z)}^{2}\right) \mu^{2}\right. \\
& \left.-3 v^{2}+2 V_{0} \mu^{2} e^{\mu w}\right) \Phi=0
\end{aligned}
$$

where $\Psi(x, y, z, \phi)=\Phi(w) \exp \left(\frac{\sqrt{3} v}{\mu} x+\mu_{(y)} y+\mu_{(z)} z\right)$, $\Phi^{\prime}=\frac{\mathrm{d} \Phi(w)}{\mathrm{d} w}$, and $w=\frac{\sqrt{3}}{\mu} x+\phi$. Hence, for various values of the constant $\mu$ from (53), we have

$$
\begin{aligned}
\Phi(w)= & \exp \left(\frac{3 \mu w}{\mu^{2}-3}\right)\left[\Phi_{1} J_{\bar{c}}\left(2 \sqrt{\frac{2 V_{0}}{\mu^{2}-3}} e^{\frac{\mu}{2} w}\right)\right. \\
& \left.+\Phi_{2} Y_{\bar{c}}\left(2 \sqrt{\frac{2 V_{0}}{\mu^{2}-3}} e^{\frac{\mu}{2} w}\right)\right], \text { with } \mu \neq \sqrt{\mathbf{3}}
\end{aligned}
$$

where $\bar{c}=\frac{2}{\left|\mu^{2}-3\right|} \sqrt{3 v^{2}-\left(\mu^{2}-3\right)\left(\mu_{(y)}^{2}+\mu_{(z)}^{2}\right)}$ and

$$
\begin{aligned}
\Phi(w)= & \Phi_{0} \exp \left[\frac{1}{2 v}\left(\mu_{(y)}^{2}+\mu_{(z)}^{2}\right)\right. \\
& \left.-\frac{v}{2} w+\frac{\sqrt{3}}{3} \frac{V_{0}}{v} e^{\sqrt{3} w}\right], \quad \text { for }|\mu|=\sqrt{3}, v \neq 0 .
\end{aligned}
$$

\subsubsection{The WKB approximation and the classical solutions}

In the WKB approximation the WDW equation (48) becomes the Hamilton-Jacobi equation

$$
\begin{aligned}
& \frac{1}{2}\left[\left(\frac{\partial S}{\partial x}\right)^{2}-\left(\frac{\partial S}{\partial y}\right)^{2}-\left(\frac{\partial S}{\partial z}\right)^{2}-\left(\frac{\partial S}{\partial \phi}\right)^{2}\right] \\
& -e^{\sqrt{3} x} V(\phi)=0
\end{aligned}
$$

where $S=S(x, y, z, \phi)$ describes a motion of a particle in the $M^{4}$ space. The solution of the Hamilton-Jacobi function leads to the following reduced Hamiltonian system:

$\dot{x}=\frac{\partial S}{\partial x}, \quad \dot{y}=\frac{\partial S}{\partial y}, \quad \dot{z}=\frac{\partial S}{\partial z}, \quad \dot{\phi}=\frac{\partial S}{\partial \phi}$.

For $V(\phi)=0$, from Eq. (56) we have that

$$
\begin{aligned}
S_{0}(x, y, z, \phi) & =c_{1} y+c_{2} z+c_{3} \phi+\varepsilon \sqrt{c_{1}^{2}+c_{2}^{2}+c_{3}^{2}} x, \\
\varepsilon & = \pm 1 .
\end{aligned}
$$


Then from (57) and (58) we have

$x(\tau)=\varepsilon \sqrt{c_{1}^{2}+c_{2}^{2}+c_{3}^{2}} t+x_{0}$.

$y(t)=-c_{1} t+y_{0}, \quad z(t)=-c_{2} t+z_{0}$,

$\phi(t)=-c_{3} t+\phi_{0}$.

Similarly, for constant potential, i.e. $V(\phi)=V_{0}$, we have

$$
\begin{aligned}
S_{V_{0}}(x, y, z, \phi)= & c_{1} y+c_{2} z+c_{3} \phi \\
& +\varepsilon \frac{2 \sqrt{3}}{3}\left(L(x)+\sqrt{c_{1-3}} \arctan h \frac{L(x)}{\sqrt{c_{1-3}}}\right)
\end{aligned}
$$

where $L(x)=\sqrt{c_{1}^{2}+c_{2}^{2}+c_{3}^{2}+2 V_{0} e^{\sqrt{3} x}}$ and $c_{1-3}=c_{1}^{2}+$ $c_{2}^{2}+c_{3}^{2}$. Hence the reduced Hamilton equations (57) are

$\dot{x}=L(x), \quad \dot{y}=-c_{1}, \quad \dot{z}=-c_{2}, \quad \phi=-c_{3}$,

and the exact solution of the field equations is

$x(t)=\frac{1}{3} \ln \left[\frac{c_{1-3}}{2 V_{0}}\left(\tanh \left(\frac{\sqrt{3}}{2} \sqrt{c_{1-3}}\left(t+x_{0}\right)\right)-1\right)\right]$,

$y(t)=c_{1} t+y_{0}, z(t)=c_{2} t+z_{0}, \phi(t)=c_{3} t+\phi_{0}$.

For the exponential potential, $V(\phi)=V_{0} e^{\mu \phi}$, as we saw previously, there exist different solutions of the WDW equation for different values of the constant $\mu$. Hence, the solution of the Hamilton-Jacobi equation (56) is determined by the various values of $\mu$.

Let us set $\mu=-\sqrt{3}$. Applying the coordinate transformation $\phi=\psi+x$ in the Hamiltonian system, the new Hamilton-Jacobi equation is

$$
\begin{aligned}
& \frac{1}{2}\left[\left(\frac{\partial S}{\partial x}\right)^{2}-2\left(\frac{\partial S}{\partial x}\right)\left(\frac{\partial S}{\partial \psi}\right)-\left(\frac{\partial S}{\partial y}\right)^{2}-\left(\frac{\partial S}{\partial z}\right)^{2}\right] \\
& \quad-V_{0} e^{-\sqrt{3} \psi}=0
\end{aligned}
$$

and the reduced Hamiltonian system is

$$
\begin{aligned}
\dot{x} & =\left(\frac{\partial S}{\partial x}\right)-\left(\frac{\partial S}{\partial \psi}\right), \quad \dot{y}=-\frac{\partial S}{\partial y}, \quad \dot{z}=-\frac{\partial S}{\partial z}, \\
\dot{\psi} & =-\frac{\partial S}{\partial x} .
\end{aligned}
$$

Therefore from (65) the Hamilton action is

$$
\begin{aligned}
S(x, y, z, \psi)= & c_{1} x+c_{2} y+c_{3} z+\frac{\left(c_{2}^{2}+c_{3}^{2}-c_{1}^{2}\right)}{2 c_{1}} \psi \\
& -\frac{\sqrt{3} V_{0}}{6 c_{1}} e^{-\sqrt{3} \psi}
\end{aligned}
$$

and the field equations reduce to the system

$$
\begin{aligned}
& \dot{x}=\frac{\left(c_{1}^{2}-c_{2}^{2}-c_{3}^{2}\right)-V_{0} e^{\sqrt{3} \psi}}{2 c_{1}}, \quad \dot{y}=-c_{2}, \quad \dot{z}=-c_{3}, \\
& \dot{\psi}=-c_{1},
\end{aligned}
$$

with the solution

$y(t)=-c_{2} t+y_{0}, \quad z(t)=-c_{3} t+z_{0}$,

$\psi(t)=-c_{1} t+\psi_{0}$,

and

$x(t)=\frac{3}{2} c_{1} t-\frac{\left(c_{2}^{2}+c_{3}^{2}\right)}{2 c_{1}} t+\frac{\sqrt{3} V_{0}}{6 c_{1}^{2}} e^{-\sqrt{3} \psi_{0}} e^{\sqrt{3} c_{1} t}+x_{0}$.

Furthermore, for $|\mu| \neq \sqrt{3}$, we apply the coordinate transformation $\phi=\psi-\frac{\sqrt{3}}{\mu} x$,

$$
\begin{aligned}
& \frac{1}{2}\left[\left(\frac{\partial S}{\partial x}\right)^{2}-\frac{2 \sqrt{3}}{\mu}\left(\frac{\partial S}{\partial x}\right)\left(\frac{\partial S}{\partial \psi}\right)-\left(1-\frac{3}{\mu^{2}}\right)\left(\frac{\partial S}{\partial \psi}\right)^{2}\right. \\
& \left.-\left(\frac{\partial S}{\partial y}\right)^{2}-\left(\frac{\partial S}{\partial z}\right)^{2}\right]-V_{0} e^{\mu \psi}=0
\end{aligned}
$$

hence from the Hamilton-Jacobi equation (56) we have

$S(x, y, z, \psi)=c_{1} x+c_{2} y+c_{3} z+\gamma(\psi)$

where

$$
\left(\frac{\mathrm{d} \gamma}{\mathrm{d} \psi}\right)^{2}=\frac{\mu^{2}}{\mu^{2}-3}\left(\frac{2 \sqrt{3}}{\mu} \frac{\mathrm{d} \gamma}{\mathrm{d} \psi}-V_{0} e^{\mu \psi}+\left(c_{1}^{2}-c_{2}^{2}-c_{3}^{2}\right)\right) .
$$

The reduced Hamiltonian system (57) in the new coordinates becomes

$\dot{y}=-\frac{\partial S}{\partial y}, \quad \dot{z}=-\frac{\partial S}{\partial z}$,

$\dot{x}=\left(\frac{\partial S}{\partial x}\right)-\frac{\sqrt{3}}{\mu}\left(\frac{\partial S}{\partial \psi}\right)$,

$\dot{\psi}=-\frac{\sqrt{3}}{\mu}\left(\frac{\partial S}{\partial x}\right)-\left(1-\frac{3}{\mu^{2}}\right)\left(\frac{\partial S}{\partial \psi}\right)$

and therefore from (72) and (73) the last equation becomes

$\dot{y}=-c_{2}, \quad \dot{z}=-c_{3}$,

$\dot{x}=c_{1}-\frac{3-\sqrt{3} \sqrt{\left(3-\mu^{2}\right) c_{1-3} V_{0} e^{\mu \psi}}}{\mu^{2}-3}$,

$\dot{y}=-\frac{2 \sqrt{3}}{\mu} c_{1}-\frac{1}{\mu} \sqrt{\left(3-\mu^{2}\right) c_{1-3} V_{0} e^{\mu \psi}}$. 
However, if one wants to write an analytical solution of this system, we have to perform another transformation that is $\mathrm{d} t \rightarrow \mathrm{d} \tau$. The exact solution of the exponential potential in Bianchi I scalar-field cosmology was found in [5] so we will omit the derivation in this work.

\subsection{Bianchi II cosmology}

One can observe from Tables 3 and 4 that, for Bianchi type II spacetimes, we can determine invariant solution of the WDW equation only for zero potential, i.e. the scalar field is a kination fluid acting as stiff matter $p_{\phi}=\rho_{\phi}$. In this case, the WDW equation (47) becomes

$\Psi_{, x x}-\Psi_{, y y}-\Psi_{, z z}-\Psi_{, \phi \phi}+e^{\frac{2 \sqrt{3}}{3}(2 y+x)} \Psi=0$.

By applying the zeroth-order invariants of the following three dimensional closed Lie algebra with vanishing commutators:

$K_{(x)}-\frac{1}{2} K_{(y)}+v \Psi \partial_{\Psi}, K_{(z)}+\mu_{(z)} \Psi \partial_{\Psi}, K_{(\phi)}+\mu_{(\phi)} \Psi \partial_{\Psi}$

we find the invariant solution

$$
\begin{aligned}
\Psi_{1}(x, y, z, \phi)= & \exp \left(\frac{2 v}{3}(y+2 x)+\mu_{(z)} z+\mu_{(\phi)} \phi\right) \\
& \left(\Psi_{1} I_{\lambda}(u(x, y))+\Psi_{2} K_{\lambda}(u(x, y))\right)
\end{aligned}
$$

where $\lambda=\frac{1}{3} \sqrt{12 v^{2}-9\left(\mu_{(z)}^{2}+\mu_{(\phi)}^{2}\right)}$ and $u(x, y)=\exp$ $\left(\frac{\sqrt{3}}{3}(2 y+x)\right)$.

One can also consider the Lie algebra $\left\{K_{(z)}, K_{(x)}-\frac{1}{2} K_{(y)}\right.$, $\left.R_{(x z)}-\frac{1}{2} R_{(y z)}\right\}$ for which we have the invariant solution

$$
\begin{aligned}
\Psi_{2}(x, y, z, \phi)= & \left(\Psi_{3} e^{c_{1} \phi}+\Psi_{4} e^{-c_{1} \phi}\right)\left(\Psi_{1} I_{i c_{1}}(u(x, y))\right. \\
& \left.+\Psi_{2} K_{i c_{1}}(u(x, y))\right)
\end{aligned}
$$

whereas, for the Lie algebra $\left\{K_{(\phi)}, K_{(x)}-\frac{1}{2} K_{(y)}, R_{(x \phi)}-\frac{1}{2}\right.$ $\left.R_{(y \phi)}\right\}$, the invariant solution is

$$
\begin{aligned}
\Psi_{3}(x, y, z, \phi)= & \left(\Psi_{3} e^{c_{1} z}+\Psi_{4} e^{-c_{1} z}\right)\left(\Psi_{1} I_{i c_{1}}(u(x, y))\right. \\
& \left.+\Psi_{2} K_{i c_{1}}(u(x, y))\right) .
\end{aligned}
$$

The Lie algebras $\left\{R_{(z \phi)}, K_{(x)}-\frac{1}{2} K_{(y)}, R_{(x \phi)}-\frac{1}{2} R_{(y \phi)}\right\}$ and $\left\{R_{(z \phi)}, K_{(x)}-\frac{1}{2} K_{(y)}, R_{(x z)}-\frac{1}{2} R_{(y z)}\right\}$ provide the solution of the form

$$
\Psi_{4}(x, y, z, \phi)=\Psi_{1} I_{0}(u(x, y))+\Psi_{2} K_{0}(u(x, y)) .
$$

In the WKB approximation, where the WDW equation (79) reduces to the Hamilton-Jacobi equation, we apply the same approach as for the case of general relativity, Sect. 4.2.1, hence we will omit it. However, we would like to note that the solution for the kination scalar field is $\phi(t)=c_{\phi} t$, where $c_{\phi}$ is a constant. In the following section, we discuss how these solutions can be applied, under a conformal transformation, in the case of $f(\mathcal{R})$ hybrid gravity. This means that the approach can easily be extended to higher-order gravity and non-minimally coupled cases.

\section{Hybrid gravity in Bianchi cosmology}

In this section we consider the action of the hybrid metric-Palatini gravity with the action of the form [47-49]

$S=\frac{1}{2 \kappa^{2}} \int \mathrm{d}^{4} x \sqrt{-g}[R+f(\mathcal{R})]$,

where $R$ is the metric Ricci curvature scalar and $f(R)$ is a function of the Palatini curvature scalar which is constructed by an independent connection $\hat{\Gamma}$. A variation of the above action with respect to the metric gives the gravitational field equations

$G_{\mu \nu}+f^{\prime}(\mathcal{R}) \mathcal{R}_{\mu \nu}-\frac{1}{2} f(\mathcal{R}) g_{i j}=0$

where $G_{\mu \nu}$ is the Einstein tensor for metric $g_{i j}$ while $R_{\mu \nu}$ is the Ricci tensor constructed by the conformally related metric $h_{\mu \nu}=$ $f^{\prime}(R) g_{\mu \nu}$ [50]. It is well known that hybrid gravity is equivalent to a non-minimally coupled scalar-tensor theory [49]. In particular, if we consider a new scalar field $\psi=f^{\prime}(\mathcal{R})$ by using a Lagrange multiplier and the relation between the two Ricci scalars $R$ and $\mathcal{R}$, the action (85) can be written in the following form:

$S=\frac{1}{2 \kappa^{2}} \int \mathrm{d}^{4} x \sqrt{-g}\left[(1+\psi) R+\frac{3}{2 \psi} \partial^{\mu} \psi \partial_{\mu} \psi-V(\psi)\right]$,

where

$V(\psi)=\mathcal{R} f^{\prime}(\mathcal{R})-f(\mathcal{R})$

is a Clairaut equation with the singular solution

$\frac{\partial V\left(f^{\prime}(\mathcal{R})\right)}{\partial f^{\prime}(\mathcal{R})}-\mathcal{R}=0$

Furthermore, from the action (87) and for the Bianchi spacetimes (1), we see that the Lagrangian of the field equations is

$$
\begin{aligned}
L_{f(\mathcal{R})=} & N(t) e^{3 \lambda} \\
& \times\left[(1+\psi)\left(6 \dot{\lambda}^{2}-\frac{3}{2}\left(\dot{\beta}_{1}^{2}+\dot{\beta}_{2}^{2}\right)\right)+6 \dot{\lambda} \dot{\psi}+\frac{3}{2 \psi} \dot{\psi}^{2}\right] \\
& +\frac{e^{3 \lambda}}{N(t)}\left(V(\psi)+(1+\psi) R^{*}\right) .
\end{aligned}
$$

As shown in [29], the action (87) of the hybrid gravity is equivalent to a phantom minimally coupled scalar field under the conformal transformation $\bar{g}_{i j}=(1+\psi) g_{i j}$, and the action (87) becomes

$$
\begin{aligned}
S= & \int \mathrm{d}^{4} x \sqrt{-g} \\
& \times\left[\bar{R}+\frac{3}{2}\left(\frac{2 \phi+3}{2(1+\phi) \phi}\right) g^{i j} \psi_{; i} \psi_{; j}-\frac{1}{(1+\psi)^{2}} V(\psi)\right]
\end{aligned}
$$

where $\bar{R}$ is the Ricci scalar of the conformal metric $\bar{g}_{i j}$; therefore under the transformation $\mathrm{d} \Phi=i \sqrt{\left(\frac{6 \psi+9}{2(1+\psi) \psi}\right)} \mathrm{d} \psi$ and $\bar{V}(\phi)=$ $-\frac{1}{(1+\psi)^{2}} V(\psi)$, we have the action of the form of (40). From the 
transformation $\psi \rightarrow \phi$, we find that the only potential which admits Lie point symmetries has the following form:

$V(\psi)=V_{0}(1+\psi)^{2} \exp (-\kappa \arctan \sqrt{\psi})$

which is the exponential potential in the case of minimally coupled scalar-field cosmology with $\kappa=\kappa(\mu)$ and $\kappa(0)=0$, i.e. $V(\psi)=$ $V_{0}(1+\psi)^{2}$. This potential transforms to the constant potential in the case of minimally coupled scalar field [55].

\section{Discussion and conclusions}

In this work we studied the Lie symmetries of the WDW equation in the Bianchi Class A spacetimes for general relativity and scalar field cosmologies, considering minimally coupled scalar-tensor gravity and non-minimally coupled gravity coming from Hybrid Gravity. We applied the Lie invariants in order to construct solutions of the WDW equation. In the case of general relativity, we found exact solutions of the WDW equation for the Bianchi I and Bianchi II spacetimes. In scalar-field cosmology, we applied the Lie symmetries as a criterion for the selection of the unknown potential of the scalar field and we were able to construct exact solutions for the Bianchi I spacetime for zero potential $V(\phi)=0$, constant potential $V(\phi)=V_{0}$, and exponential potential $V(\phi)=e^{\mu \phi}$. For the Bianchi II spacetime we obtained solutions for the zero potential case. In each case, we show that when the WDW equation is invariant under the action of the three dimensional Lie algebra with zero commutators, the Hamilton-Jacobi equation of the Hamiltonian system, which was defined by the field equations, can be solved by the method of separation of variables; that means that the field equations are Liouville integrable. It is important to note that, in the case of FLRW scalar cosmology, we have more potentials where the WDW admits Lie symmetries. However, since the Lie symmetries are connected to the conformal algebra of the minisuperspace, in the case of FLRW scalar-field cosmology, the dimension of the minisuperspace is two, which means that the last admits an infinite number of conformal killing vectors, whereas, for the Bianchi models, the minisuperspace has dimension four and admits a 15 dimensional conformal algebra, i.e. less possible generators for the Lie symmetries of the WDW equation.

Finally, we studied the case of the hybrid gravity in the Bianchi Class A spacetimes. Since hybrid gravity is equivalent to a scalartensor theory, we were able to related all the potentials we found in the case of minimally coupled scalar field to that of hybrid gravity.

This analysis is important in the sense that can be used in order to construct solutions of the wave function of the Universe and, at the same time, conservation laws, and classical solutions for the field equations. Following the discussion in [56], the presence of symmetries gives rise to a straightforward interpretation of the Hartle criterion: the symmetries generates oscillatory behaviors in the wave function of the Universe and then allow correlations among physical variables. This give rise to classically observable cosmological solutions. Here we generalized this result considering Bianchi models. On the other hand, other general selection rules can be identified in quantum cosmology, as discussed in [57]. This will be the topic of forthcoming papers.

A final remark is now in order concerning the possible applications of the above solutions. In general, the solutions of the WDW equation can be used to construct/determine the quantum potential
$[24,35,58,59]$ in the semi-classical approach of Bohmian mechanics $[60,61]$. Specifically, if the solution of the WDW equation is $\Psi\left(x^{k}\right)=A\left(x^{k}\right) e^{i B\left(x^{k}\right)}$, where $A\left(x^{k}\right)$ is not, in general, a slowroll function, then substituting this solution in the WDW equation gives

$H\left(p_{k}, x^{k}\right)+Q_{V}=0$,

where $H\left(p_{k}, x^{k}\right)$ is the Hamiltonian function which generates the WDW equation. Starting from our considerations in the lhs of Eqs. (15) and (45), $Q_{V}=\frac{\Delta(A)}{A}$, is the quantum potential and $p_{\mu}=\frac{\partial B\left(x^{k}\right)}{\partial x^{\mu}}$. Equation (93) is the new Hamiltonian of the field equations providing the semi-classical solution.

Furthermore, in the limit $Q_{V} \rightarrow 0$, or $A$ is a slow-roll function, we are in the WKB approximation if and only if $B\left(x^{k}\right)$ is a solution of the related Hamilton-Jacobi equation. Let us consider now the wavefunction (21) for the Bianchi I spacetime, where we can see that, for $\mu_{1}, \mu_{2} \in I$, the quantum potential is zero. Since the corresponding function $B\left(x^{k}\right)$ is the classical action integral, this wavefunction leads to a Kasner Universe as discussed in Sect. 4.1.

On the other hand, for the wavefunction (22), where $\mu_{1}, \mu \in I^{*}$, and for large values of $\sqrt{y^{2}+z^{2}}$, due to the anisotropic parameters $\beta_{1}, \beta_{2}$, the wavefunction (22) can be approximated by

$$
\begin{aligned}
\Psi_{I}(x, y, z) \simeq & \frac{\cos \left(\sqrt{y^{2}+z^{2}}\right)}{\left(y^{2}+z^{2}\right)^{\frac{1}{4}}} \\
& \times \exp \left(i\left(\operatorname{Im}(\mu) \arctan \frac{y}{z}+\operatorname{Im}\left(\mu_{1}\right) x\right)\right)
\end{aligned}
$$

and then the quantum potential is $Q_{V}=1-\frac{1}{4}\left(x^{2}+y^{2}\right)^{-1}$. The semi-classical field equations are

$\dot{x}=\operatorname{Im}\left(\mu_{1}\right), \quad \dot{y}=\frac{\operatorname{Im}(\mu)}{y^{2}+z^{2}}, \quad \dot{z}=-\frac{\operatorname{Im}(\mu)}{y^{2}+z^{2}}$

or by selecting the new variables $y=r \cos \theta, z=r \sin \theta$, we find that $\dot{r}=0$, that is, $r=r_{0}$, and $\theta=-\frac{\operatorname{Im}(\mu)}{r_{0}^{2}} t+\theta_{0}$, where $r_{0}$ is large. Hence

$$
\begin{aligned}
& x \simeq t, \quad y \simeq r_{0} \cos \left(-\frac{\operatorname{Im}(\mu)}{r_{0}^{2}} t+\theta_{0}\right), \\
& z \simeq r_{0} \sin \left(-\frac{\operatorname{Im}(\mu)}{r_{0}^{2}} t+\theta_{0}\right),
\end{aligned}
$$

which differs from the above Kasner solution (26).

In a forthcoming work we will extend our analysis and we study the difference of the semi-classical solutions arising from the group invariant solutions of the WDW equation.

Acknowledgments AP acknowledges financial support of FONDECYT Grant No. 3160121. AW acknowledges financial support from 1445/M/IFT/15 and DEC-2013/09/B/ST2/03455. SC acknowledges the support of INFN (iniziative specifiche QGSKY and TEONGRAV).

Open Access This article is distributed under the terms of the Creative Commons Attribution 4.0 International License (http://creativecomm ons.org/licenses/by/4.0/), which permits unrestricted use, distribution, 
and reproduction in any medium, provided you give appropriate credit to the original author(s) and the source, provide a link to the Creative Commons license, and indicate if changes were made.

Funded by SCOAP ${ }^{3}$.

\section{Appendix A: Symmetries of differential equations and invariant functions}

In this appendix, we briefly discuss the basic properties and definitions of the symmetries of differential equations (DEs). Furthermore we discuss the application of the Lie symmetries of the WDW equation showing that if the WDW equation admits Lie symmetries, which form a Lie algebra with zero commutators, then the WDW equation admits oscillatory terms in the solution as many as the dimension of the minisuperspace and, at the same time, the Hamiltonian system which defined by the field equations is Liouville integrable; that is, the field equations can be solved by quadratures. In such a case, classical cosmological solutions can be derived.

Lie point symmetries and invariant functions

A second-order DE is a function $H=H\left(x^{i}, u^{A}, u_{, i}^{A}, u_{, i j}^{A}\right)$ in the jet space $B_{M}$, where $x^{i}$ are the independent variables and $u^{A}$ are the dependent ones. Let

$\mathbf{X}=\xi^{i}\left(x^{k}, u^{B}\right) \partial_{x^{i}}+\eta^{A}\left(x^{k}, u^{B}\right) \partial_{u^{A}}$.

be the generator of the infinitesimal point transformation

$$
\begin{aligned}
\bar{x}^{i} & =x^{i}+\varepsilon \xi^{i}\left(x^{k}, u^{B}\right), \\
\bar{u}^{A} & =\bar{u}^{A}+\varepsilon \eta^{A}\left(x^{k}, u^{B}\right) .
\end{aligned}
$$

The function $H=0$, is invariant under the action of the infinitesimal point transformation (A2), (A3) if there exists a function $\lambda$ such that [51]

$\mathbf{X}^{[2]}(H)=\lambda H$.

The vector field $X$ is called Lie point symmetry of the function $H$ and $X^{[n]}$ is the second prolongation of $X$ in the jet space $B_{M}$

$\mathbf{X}^{[2]}=\mathbf{X}+\eta_{i}^{A} \partial_{\dot{x}^{i}}+\eta_{i j}^{A} \partial_{u_{i j}^{A}}$,

where

$\eta_{i}^{A}=\eta_{, i}^{A}+u_{, i}^{B} \eta_{, B}^{A}-\xi_{, i}^{j} u_{, j}^{A}-u_{, i}^{A} u_{, j}^{B} \xi_{, B}^{j}$

and

$$
\begin{aligned}
\eta_{i j}^{A}= & \eta_{, i j}^{A}+2 \eta_{, B(i}^{A} u_{, j)}^{B}-\xi_{, i j}^{k} u_{, k}^{A}+\eta_{, B C}^{A} u_{, i}^{B} u_{, j}^{C} \\
& -2 \xi_{, i|B|}^{k} u_{j)}^{B} u_{, k}^{A}-\xi_{, B C}^{k} u_{, i}^{B} u_{, j}^{C} u_{, k}^{A}+\eta_{, B}^{A} u_{, i j}^{B}-2 \xi_{,(j}^{k} u_{, i) k}^{A} \\
& -\xi_{, B}^{k}\left(u_{, k}^{A} u_{, i j}^{B}+2 u_{(, j}^{B} u_{, i) k}^{A}\right) .
\end{aligned}
$$

The importance of Lie point symmetries is that they can be used in order to reduce an order of a differential equation. When a reduction is possible, one can determine invariant solutions or transform them to other ones [52]. From condition (A4) one defines the Lagrange system $\frac{\mathrm{d} x^{i}}{\xi^{i}}=\frac{\mathrm{d} u}{\eta}=\frac{\mathrm{d} u_{i}}{\eta_{[i]}}=\frac{\mathrm{d} u_{i j}}{\eta_{i j}}$,

whose solution provides the characteristic functions

$$
Z^{[0]}\left(x^{k}, u\right), Z^{[1] i}\left(x^{k}, u, u_{i}\right), Z^{[2]}\left(x^{k}, u, u_{, i}, u_{i j}\right) .
$$

The solution $Z^{[k]}$ is called the $k$ th-order invariant of the Lie symmetry vector (A1). By writing the DE in terms of the invariants $Z^{[k]}$, we can reduce the order of the DE; for details see for instance $[52,53]$. Below we discuss the application of the Lie symmetries and of the Lie invariants for the WDW equation.

Reduction and invariant solutions of the WDW equation by Lie point symmetries

In order to determine the Lie symmetries of the WDW equation we apply a geometric method which is established by Paliathanasis and Tsamparlis [7]. The method relates the Lie symmetries of the Klein-Gordon equation to the conformal algebra of the underlying geometry. Hence, in the following we will not present the construction of the Lie symmetries of the WDW equation but we will give the results.

In particular, the general form of a Lie symmetry of the WDW equation is

$\mathbf{X}=\xi^{i}\left(x^{k}\right) \partial_{i}+\left(\frac{2-n}{2} \psi \Psi+a_{0} \Psi\right) \partial_{\Psi}$,

where $\xi^{i}\left(x^{k}\right)$ is a CKV of the metric which defines the conformal Laplace operator, (in our consideration the minisuperspace) and $\psi\left(x^{k}\right)$ is the conformal factor of the CKV, recall that since $\xi^{i}$ is a CKV of $g_{i j}$, it means that $L_{\xi} g_{i j}=2 \psi g_{i j}$.

Furthermore, it is possible to consider a coordinate transformation $x^{i} \rightarrow \bar{x}^{i}$ so that $\xi^{i}\left(x^{k}\right) \partial_{i} \rightarrow \partial_{J} \quad$ (these are called normal coordinates). In the normal coordinates the symmetry vector takes the following simple form:

$\mathbf{X}=\partial_{J}+\left(\frac{2-n}{2} \psi \Psi+a_{0} \Psi\right) \partial_{u}$

where now either with the method of Lie invariants, or with the method of linear differential operators (see [33] for details) we find the following expression for the solution of $\Psi$ :

$\Psi\left(\bar{x}^{b}, \bar{x}^{J}\right)=\Phi\left(\bar{x}^{b}\right) \exp \left[\int\left(\frac{2-n}{2} \psi-Q_{0}\right) d \bar{x}^{J}\right]$,

from which follows again that the coordinate $x^{J}$ is factored out from the solution $\Psi$.

Furthermore, in [7] it was also shown that the symmetries of the WDW equation can be used in order to find Noether symmetries for classical particles. ${ }^{3}$ The exact relation among the Lie symmetries of the WDW equation, the Noetherian conservation laws of the field equations, and the interpretability conditions is given in [33].

\footnotetext{
3 A similar methodology by using the variational symmetries has been established recently in [54].
} 


\section{References}

1. E.W. Kolb, M.S. Turner, The early universe (Addison-Wesley, New York, 1990)

2. C.W. Misner, The Isotropy of the universe. Astrophys. J. 151, 431 (1968)

3. O. Hrycyna, M. Szydlowski, Dynamics of the Bianchi I model with non-minimally coupled scalar field near the singularity. AIP Conf. Proc. 1514, 191 (2013)

4. E. Russel, C. Battal Kilinc, O.K Pashaev, Bianch I Model: an alternative way to model the Presentday Universe. arXiv:1312.3502v3

5. M. Tsamparlis, A. Paliathanasis, The geometric nature of Lie and Noether symmetries. Gen. Relat. Gravit. 43, 1861 (2011)

6. Yu Al Kamenshchik, E.O. Pozdeeva, A. Tronconi, G. Venturi, S.Yu. Vernov, Integrable cosmological models with non-minimally coupled scalar fields. Class. Quant. Grav. 31, 105003 (2014)

7. A. Paliathanasis, M. Tsamparlis, The geometric origin of Lie point symmetries of the Schrodinger and the Klein Gordon equations. Int. J. Geom. Methods Mod. Phys. 11, 1450037 (2014)

8. Y. Zhang, Y.G. Gong, Z.H. Zhu, Noether symmetry approach in multiple scalar fields scenario. Phys. Lett. B 688, 13 (2010)

9. S. Basilakos, M. Tsamparlis, A. Paliathanasis, Using the Noether symmetry approach to probe the nature of dark energy. Phys. Rev. D 83, $103512(2011)$

10. Y. Kucukakca, U. Camci, I. Semiz, LRS Bianchi type I universes exhibiting Noether symmetry in the scalar-tensor Brans-Dicke theory. Gen. Rel. Gravit. 44, 1893 (2012)

11. B. Vakili, F. Khazaie, Noether symmetric classical and quantum scalar field cosmology. Class. Quant. Grav. 29, 035015 (2012)

12. R.C. de Souza, R. Andre, G.M. Kremer, Analysis of the nonminimally coupled scalar field in the Palatini formalism by the Noether symmetry approach. Phys. Rev. D 87, 083510 (2013)

13. S. Capozziello, R. de Ritis, P. Scudellaro, Noether's symmetries in $(\mathrm{n}+1)$-dimensional nonminimally coupled cosmologies. Int. J. Mod. Phys. D 2, 465 (1993)

14. S. Capozziello, E. Piedipalumbo, C. Rubano, P. Scudellaro, Noether symmetry approach in phantom quintessence cosmology. Phys. Rev. D. 80, 104030 (2009)

15. A. Aslam, M. Jabil, D. Momeni, R. Myrzakulov, M.A. Rashid, M. Raza, Noether gauge symmetry approach in quintom cosmology. Astrophys. Space Sci. 348, 533 (2013)

16. A. Paliathanasis, M. Tsamparlis, S. Basilakos, Constraints and analytical solutions of $f(R)$ theories of gravity using Noether symmetries. Phys. Rev. D. 84, 123514 (2011)

17. B. Vakili, Noether symmetric $f(R)$ quantum cosmology and its classical correlations. Phys. Lett. B 669, 209 (2008)

18. N. Dimakis, T. Christodoulakis, P.A. Terzis, FLRW metric f(R) cosmology with a perfect fluid by generating integrals of motion. J. Geom. Phys. 77, 97 (2014)

19. S. Capozziello, G. Lambiase, Higher order corrections to the effective gravitational action from Noether symmetry approach. Gen. Relativ. Gravit. 32, 29 (1999)

20. S. Capozziello, A. De Felice, $f(R)$ cosmology by Noether's symmetry. JCAP 0808, 016 (2008)

21. M. Tsamparlis, A. Paliathanasis, Generalizing the autonomous Kepler-Ermakov system in a Riemannian space. J. Phys. A $\mathbf{4 5}$, 275202 (2012)

22. S. Capozziello, N. Frusciante, D. Vernieri, New Spherically Symmetric Solutions in $\mathrm{f}(\mathrm{R})$-gravity by Noether Symmetries. Gen. Rel. Gravit. 44, 1881 (2012)

23. A. Paliathanasis, S. Basilakos, E.N. Saridakis, S. Capozziello, K. Atazadeh, F. Darabi, M. Tsamparlis, New Schwarzschild-like solutions in $\mathrm{f}(\mathrm{T})$ gravity through Noether symmetries. Phys. Rev. D 89, 104042 (2014)
24. T. Christodoulakis, N. Dimakis, P.A. Terzis, B. Vakili, E. Melas, T. Grammenos, Minisuperspace canonical quantization of the Reissner-Nordström black hole via conditional symmetries, Phys.Rev. D 89(4), 044031 (2014)

25. T. Christodoulakis, N. Dimakis, P.A. Terzis, Th Grammenos, E. Melas, A. Spanou, Conditional Symmetries and the Canonical Quantization of Constrained Minisuperspace Actions: the Schwarzschild case. J. Geom. Phys. 71, 127 (2013)

26. S. Basilakos, S. Capozziello, M. De Laurentis, A. Paliathanasis, M. Tsamparlis, Noether symmetries and analytical solutions in $\mathrm{f}(\mathrm{T})$ cosmology: a complete study. Phys. Rev. D 88, 103526 (2013)

27. H. Wei, X.J. Guo, L.F. Wang, Noether Symmetry in $\mathrm{f}(\mathrm{T})$ Theory. Phys. Lett. B 707, 298 (2012)

28. Y. Kucukakca, Teleparallel dark energy model with a fermionic field via Noether symmetry, Eur. Phys. J. C 74(10), 3086 (2014)

29. A. Borowiec, S. Capozziello, M. De Laurentis, F.S.N. Lobo, A. Paliathanasis, M. Paolella, A. Wojnar, Invariant solutions and Noether symmetries in Hybrid Gravity. Phys. Rev. D 91, 023517 (2015)

30. S. Capozzielo, G. Marmo, C. Rubano, P. Scudellaro, Noether symmetries in Bianchi universes. Int. J. Mod. Phys. D. 6, 491 (1997)

31. S. Cotsakis, P.G.L. Leach, H. Pantazi, Symmetries of homogeneous cosmologies. Gravit. Cosm. 4, 314 (1998)

32. B. Vakil, N. Khosvravi, H.R. Sepangi, Bianchi spacetimes in noncommutative phase space. Class. Quantum Grav. 24, 931 (2007)

33. A. Paliathanasis, M. Tsamparlis, S. Basilakos, J.D. Barrow, Dynamical analysis in scalar field cosmology. Phys. Rev. D 91, 123535 (2015)

34. A. Paliathanasis, M. Tsamparlis, S. Basilakos, J.D. Barrow, Classical and quantum solutions in Brans-Dicke cosmology with a perfect fluid. Phys. Rev. D 93, 043528 (2016)

35. A. Zampeli, T. Pailas, P.A. Terzis, T. Christodoulakis, Conditional symmetries in axisymmetric quantum cosmologies with scalar fields and the rate of the classical singularities. arXiv:1511.08382

36. M. Demianski, R. de Ritis, C. Rubano, P. Scudellaro, Scalar fields and anisotropy in cosmological models. Phys. Rev. D 46, 1391 (1992)

37. F. Hoyle, J.V. Narlikar, Proc. R. Soc. A 273, 1 (1963)

38. P.E. Hydon, Symmetry methods for differential equations: a beginner's guide, vol. 22 (Cambridge University Press, Cambridge, 2000)

39. K.C. Jacobs, Spatially homogeneous and euclidean cosmological models with shear. Astrophys. J. 153, 661 (1968)

40. J.D. Barrow, J. Stein-Schabes, Inhomogeneous cosmologies with cosmological constant. Phys. Lett. A 103, 315 (1984)

41. M.P.Jr. Rayan, L.C. Shepley, Homogeneous relativistic cosmologies (Princeton University Press, Princeton, 1975)

42. C.W. Misner, Quantum cosmology I. Phys. Rev. D 186, 1319 (1969)

43. M.A.H. MacCallumn, General relativity: an einstein centenary survey, ed. by W.S.W. Hawking, W. Israel (Cambridge University Press, Cambridge, 1979)

44. S. Capozziello, G. Marmo, C. Rubano, P. Scudellaro, Noether symmetries in Bianchi universes. Int. J. Mod. Phys. D 6, 491 (1997)

45. B. Abraham-Shrauner, K.S. Govinder, D.J. Arrigo, Type-II hidden symmetries of the linear 2D and 3D wave equations. J. Phys. A 39, 5739 (2006)

46. A. Paliathanasis, M. Tsamparlis, The reduction of the Laplace equation in certain Riemannian spaces and the resulting Type II hidden symmetries. J. Geom. Phys. 76, 107 (2014)

47. T. Harko, T.S. Koivisto, F.S.N. Lobo, G.J. Olmo, Metric-Palatini gravity unifying local constraints and late-time cosmic acceleration. Phys. Rev. D 85, 084016 (2012)

48. S. Capozziello, T. Harko, F.S.N. Lobo, G.J. Olmo, Hybrid modified gravity unifying local tests, galactic dynamics and late-time cosmic acceleration. Int. J. Mod. Phys. D 22, 1342006 (2013) 
49. S. Capozziello, T. Harko, T.S. Koivisto, F.S.N. Lobo, G.J. Olmo, Cosmology of hybrid metric-Palatini $f(X)$ gravity. JCAP 04, 011 (2013)

50. S. Capozziello, M. De Laurentis, Extended theories of gravity. Phys. Rept. 509, 167 (2011)

51. S.M. Lie, Differentialgleichungen (Chelsea, New York, 1967)

52. G.W. Bluman, S. Kumei, Symmetries of differential equations (Springer, New York, 1989)

53. H. Stephani, Differential equations: their solutions using symmetry (Cambridge University Press, New York, 1989)

54. T. Christodoulakis, N. Dimakis, P.A. Terzis, Lie point and variational symmetries in minisuperspace Einstein gravity. J. Phys. A 47, 095202 (2014)

55. S. Capozziello, R. de Ritis, A.A. Marino, Some aspects of the cosmological conformal equivalence between 'Jordan frame' and 'Einstein frame'. Class. Quant. Grav. 14, 3243 (1997)
56. S. Capozziello, M. De Laurentis, S.D. Odintsov, Hamiltonian dynamics and Noether symmetries in extended gravity cosmology. Eur. Phys. J. C 72, 2068 (2012)

57. A.O. Barvinsky, A.Yu. Kamenshchik, Selection rules for the Wheeler-DeWitt equation in quantum cosmology, Phys.Rev. D 89(4), 043526 (2014)

58. S. Goldstein, W. Struyve, J. Phys. A 48, 025303 (2015)

59. F. Tovar Falciano, N. Pinto-Neto, W. Struyve, Phys. Rev. D 91, 043524 (2015)

60. D. Bohm, Phys. Rev. 55, 166 (1952a)

61. D. Bohm, Phys. Rev. 85, 180 (1952b) 